\title{
FUNGICIDAS, DOSES E VOLUMES DE CALDA NO CONTROLE QUÍMICO DA FERRUGEM DA FOLHA DA AVEIA (Puccinia coronata f. sp. avenae)
}

\author{
ANA R. DE OLIVEIRA ${ }^{1}$, WALTER BOLLER ${ }^{2}$, CARLOS A. FORCELINI ${ }^{3}$, RUBENS BLUM ${ }^{4}$, \\ ANDRÉ LOPES ${ }^{5}$
}

\begin{abstract}
RESUMO: A ferrugem da folha (Puccinia coronata f. sp. avenae) é a doença mais destrutiva da aveia, e aplicações de fungicidas com volumes baixos de calda podem reduzir a eficácia do controle químico. O objetivo do trabalho foi avaliar a eficiência técnica e econômica de fungicidas, doses e volumes de calda no controle da ferrugem da folha da aveia. O experimento foi conduzido no ano de 2003, na área experimental da FAMV/UPF, com a cultivar de aveia UPFA-20. Os tratamentos foram compostos pelas combinações entre dois fungicidas (tebuconazole, Folicur, $0,75 \mathrm{~L} \mathrm{ha}^{-1}$ e epoxiconazole + piraclostrobim, Opera, 0,5 $\left.\mathrm{L} \mathrm{ha}^{-1}\right)$, quatro doses $(40 ; 60 ; 80$ e $100 \%$ da dose recomendada) e dois volumes de calda (100 e $\left.200 \mathrm{~L} \mathrm{ha}^{-1}\right)$. O delineamento experimental foi o de blocos casualizados, com esquema fatorial $(2 \times 4 \times 2)$ e quatro repetições. Avaliaram-se a severidade, o controle da ferrugem, a massa do hectolitro, a massa de mil grãos e o rendimento de grãos, realizando-se análise econômica. $\mathrm{O}$ volume de calda de $200 \mathrm{~L} \mathrm{ha}^{-1}$ proporcionou maiores níveis de controle da doença. As aplicações dos fungicidas com volume de $200 \mathrm{~L} \mathrm{ha}^{-1}$ e meia dose ou com $100 \mathrm{~L} \mathrm{ha}^{-1}$ e dose cheia proporcionam níveis de controle da ferrugem equivalentes. O resultado econômico difere entre fungicidas e independe do volume de calda. Os efeitos de doses dependem do fungicida.
\end{abstract}

PALAVRAS-CHAVE: tecnologia de aplicação, produtos fitossanitários, análise econômica.

\section{FUNGICIDES, RATES AND SPRAY VOLUMES IN THE CHEMICAL CONTROL OF OATS CROWN RUST (Puccinia coronata f. sp. avenae)}

\begin{abstract}
Crown rust (Puccinia coronata f. sp. avenae) is the most important disease of oats in Brazil. In susceptible oat cultivars, fungicides are needed to control the disease efficiently. However, spray at low volumes may reduce fungicide performance significantly. A field experiment with the oat cultivar UPFA-20 was carried out at the FAMV/UPF to evaluate the influence of fungicides, rates, and spray volumes on the efficacy of the chemical control for crown rust. The tested treatments combined two fungicides (tebuconazole, Folicur, $0,75 \mathrm{~L} \mathrm{ha}^{-1}$; epoxiconazole + pyraclostrobin, Opera, $0,5 \mathrm{~L} \mathrm{ha}^{-1}$ ), four rates $\left(40 ; 60 ; 80\right.$ and $100 \%$ of the commercial rate), and two spray volumes (100 and $200 \mathrm{~L} \mathrm{ha}^{-1}$ ). The field plots were arranged according to a factorial block design $(2 \times 4 \times 2)$ with four replicates. The assessments included the disease severity, disease control, grain quality and grain yield. The net profit of each treatment was also determined based upon the application costs and the sale price of oats at the harvest. At the same rate, for both fungicides, the volume of $200 \mathrm{~L} \mathrm{ha}^{-1}$ provided better disease control. At this volume, the $50 \%$ rate controlled crown rust as good as the full $(100 \%)$ rate with $100 \mathrm{~L} \mathrm{ha}^{-1}$. The net profit differed between fungicides but not for spray volumes. The rate effects were fungicide dependent.
\end{abstract}

KEYWORDS: spray technology, pesticides, economical analysis.

\footnotetext{
${ }^{1}$ Eng ${ }^{\text {a }}$ Agrônoma, Mestre em Agronomia, Faculdade de Agronomia e Medicina Veterinária da Universidade de Passo Fundo, Caixa Postal 611, Passo Fundo - RS, anaritao@bol.com.br

${ }^{2}$ Engoㅡ Agrônomo, Prof. Dr., FAMV-UPF, Caixa Postal 611, Passo Fundo - RS, boller@upf.br

${ }^{3}$ Eng ${ }^{\text {o }}$ Agrônomo, PhD, FAMV-UPF, Caixa Postal 611, Passo Fundo - RS, forcelini@upf.br

${ }^{4}$ Eng ${ }^{\mathrm{O}}$ Agrônomo, MSc, Caixa Postal 611, Passo Fundo - RS, blum@upf.br

${ }^{5}$ Acadêmico do Curso de Agronomia, FAMV-UPF, bolsista de Iniciação Científica, Pibic/CNPq.

Recebido pelo Conselho Editorial em: 3-3-2005
}

Aprovado pelo Conselho Editorial em: 30-8-2006 


\section{INTRODUÇÃO}

A tecnologia de aplicação constitui-se em área de grande importância para programas de controle de doenças de plantas (AZEVEDO, 2003). Nos atuais conceitos de aplicação de defensivos, são quatro os pontos a serem considerados como fundamentais, para obter pleno êxito, tanto na preservação das colheitas, quanto na redução de ataques de pragas e patógenos: timing ou momento oportuno, cobertura, dose e segurança (MATUO, 1998; OZEKI \& KUNZ, 1998; GUEDES \& DORNELLES, 1998). Além disso, deve-se considerar, ainda, a influência dos fatores biológicos, meteorológicos e agronômicos, nem sempre previsíveis (AZEVEDO, 2003).

Nas aplicações de líquidos, em pulverização, o diâmetro das gotas determina o nível de cobertura e também estabelece o seu comportamento quanto a distância de deslocamento, deriva, penetração entre a folhagem, perda por evaporação e, conseqüentemente, a percentagem de calda, do volume total aplicado, que permanece sobre o alvo. Para fungicidas sistêmicos, são indicadas densidades de gotas de 30 a 40 gotas $\mathrm{cm}^{-2}$, com DMV de 201 a 400 m (OZEKI \& KUNZ, 1994). Produtos sistêmicos são eficazes em condições de menor cobertura do alvo biológico quando comparados com produtos com modo de ação de contato. A cobertura requerida, em combinação com o diâmetro das gotas aplicado e a superfície exposta do alvo, determina diferentes volumes de pulverização (DELGADO, 1999).

Estudos de redução de volumes nas aplicações têm sido desenvolvidos com a finalidade de diminuir custos nos tratamentos químicos. No entanto, aplicações de fungicidas com volumes de calda inferiores a $200 \mathrm{~L} \mathrm{ha}^{-1}$ podem comprometer a eficiência desses produtos no controle de doenças em cereais de inverno (SAUER, 1999). AZEVEDO (2003) relata que, na maioria das vezes, as pulverizações de fungicidas requerem melhor cobertura do alvo biológico do que os demais produtos fitossanitários. Segundo MARTINELLI (2003), a presença da ferrugem da folha da aveia no Sul do Brasil é importante fator limitante da produção, reduzindo a quantidade e a qualidade dos grãos. O controle dessa doença vem exigindo aplicações freqüentes de fungicidas, que custam, em média, US\$ 30,00 por ha por pulverização (PICININI \& FERNANDES, 1994). Essa prática permite melhores resultados técnicos em relação à produção de aveia, porém aumenta o custo de produção e diminui o retorno econômico da cultura (MARTINELLI, 2003).

Relatos de BOLLER et al. (2001) e de BRAUN et al. (2002) mostram que uma aplicação do fungicida sistêmico tebuconazole, com volumes de calda de 200 e $300 \mathrm{~L} \mathrm{ha}^{-1}$, proporcionou, respectivamente, níveis de controle de ferrugem da folha e rendimentos de grãos, semelhantes a duas aplicações com $100 \mathrm{~L} \mathrm{ha}^{-1}$ e duas com $150 \mathrm{~L} \mathrm{ha}^{-1}$. Considerando-se que em cada aplicação a dose de fungicida foi aquela recomendada para a cultura, e levando em conta o custo de cada pulverização, esses dados sugerem ser possível reduzir a dose de fungicida quando se utilizam volumes de calda mais elevados.

Este trabalho teve por objetivo avaliar a eficiência técnica e econômica dos fungicidas epoxiconazole + piraclostrobim e tebuconazole quando aplicados com diferentes doses e volumes de calda, para o controle da ferrugem da folha da aveia.

\section{MATERIAL E MÉTODOS}

O experimento foi conduzido durante o ano agrícola de 2003, na Área experimental da Faculdade de Agronomia e Medicina Veterinária da Universidade de Passo Fundo ( $28^{\circ} 15^{\prime}$ S, $52^{\circ} 24^{\prime}$ W e altitude de $687 \mathrm{~m}$ acima do nível do mar), em Latossolo Vermelho-Escuro típico, de textura argilosa. A cultura da aveia, cultivar UPFA-20, foi semeada sob sistema plantio direto, em 27 de junho de 2003. A densidade de semeadura foi de 300 sementes viáveis por $\mathrm{m}^{2}$, tratadas com o inseticida imidaclopride (Gaucho® $50 \mathrm{ml}$ p.c. $100 \mathrm{~kg}^{-1}$ ). A distribuição das sementes e a adubação foram realizadas em linhas, distribuindo-se $250 \mathrm{~kg} \mathrm{ha}^{-1}$ de fertilizante NPK da fórmula 5-20-20. Em virtude de conduzir a cultura sob restos culturais de milho, foram realizadas duas aplicações de uréia na forma granulada de 
$40 \mathrm{~kg} \mathrm{ha}^{-1} \mathrm{de} \mathrm{N}$, em cobertura. Os demais tratos culturais foram realizados de acordo com as indicações técnicas para a cultura da aveia.

Foram utilizadas 68 parcelas com dimensões de 1,36 $\mathrm{m}$ de largura por 5,0 $\mathrm{m}$ de comprimento (oito linhas espaçadas entre si em $0,17 \mathrm{~m}$ ). O delineamento experimental foi de blocos casualizados, no esquema fatorial $2 \times 4 \times 2$, com quatro repetições. $O$ experimento totalizou 16 tratamentos na parte aérea, mais uma testemunha sem aplicação de fungicidas.

Foram combinados os fungicidas tebuconazole (Folicur® $200 \mathrm{CE}$, a $0,75 \mathrm{~L} \mathrm{ha}^{-1}$ ) e expoxiconazole + piraclostrobim $\left(\right.$ Opera ${ }^{\circledR} 183 \mathrm{SE}$, a $\left.0,50 \mathrm{~L} \mathrm{ha}^{-1}\right)$, quatro doses dos produtos $(40 ; 60$; 80 e $100 \%$ da dose recomendada) e dois volumes de calda (100 e $\left.200 \mathrm{~L} \mathrm{ha}^{-1}\right)$. Os tratamentos foram aplicados em 23-9-2003 (emborrachamento) e 17-10-2003 (florescimento), com pulverizador costal pressurizado com $\mathrm{CO}_{2}$, equipado com barra portando três bicos espaçados em $0,50 \mathrm{~m}$, que foi conduzida a $0,45 \mathrm{~m}$ acima do ápice das plantas. A ponta de pulverização utilizada foi a de jato plano de uso ampliado da série Teejet XR 110015, operada à pressão de $200 \mathrm{kPa}$, produzindo gotas de categoria fina (SPRAYING SYSTEMS Co., 2003). No momento da primeira aplicação, as condições médias determinadas a 1,20 m de altura do solo (altura de condução da barra nas aplicações) foram: umidade relativa do ar de $60 \%$, temperatura do ar de $19,5{ }^{\circ} \mathrm{C}$ e velocidade do vento de $9 \mathrm{~km} \mathrm{~h}^{-1}$. Na segunda aplicação, a temperatura, a umidade relativa do ar e a velocidade do vento foram, respectivamente, de $22{ }^{\circ} \mathrm{C}, 68 \%$ e $5,5 \mathrm{~km} \mathrm{~h}^{-1}$.

A severidade da ferrugem da folha foi avaliada nos dias 24-9-2003; 9-10-2003; 17-10-2003 e 25 10-2003, coletando-se cinco plantas ao acaso por parcela e destacando-se todas as folhas totalmente expandidas. As avaliações do nível de severidade da ferrugem da folha foram realizadas com base em escala diagramática descrita por CALPOUZOS et al. (1976). Os percentuais de severidade da doença, lidos nas avaliações, foram integrados em função dos dias decorridos entre as leituras, obtendo-se a área abaixo da curva de progresso da ferrugem da folha (AACPFF), com o auxílio da eq.(1). em que,

$$
\mathrm{AACPFF}=\Sigma\left\{\left[\left(\mathrm{y}_{1}+\mathrm{y}_{2}\right) / 2\right]\left(\mathrm{t}_{2}-\mathrm{t}_{1}\right)\right\}
$$

$\mathrm{y}_{1}$ e $\mathrm{y}_{2}$ - valores de duas leituras consecutivas de severidade da ferrugem da folha, e

$\mathrm{t}_{1}$ e $\mathrm{t}_{2}$ - datas de duas leituras consecutivas de severidade da ferrugem da folha.

Comparando-se a AACPFF de cada parcela com a média das testemunhas, calcularam-se os níveis de controle da ferrugem da folha da aveia. A colheita foi realizada em 15-11-2003, e, posteriormente, os grãos foram desaristados e limpos, determinando-se a sua massa, o teor de água, a massa de mil grãos (PMG), a massa do hectolitro (PH) e calculando-se o rendimento de grãos, expresso em $\mathrm{kg} \mathrm{ha}^{-1}$, com teor de água padronizado para 13\%. Para a realização da análise econômica do experimento, foram calculados a receita bruta, a receita líquida e o resultado econômico obtido em função dos tratamentos químicos. A receita bruta foi obtida multiplicando-se o rendimento de grãos $\left(\mathrm{kg} \mathrm{ha}^{-1}\right)$ pelo preço de mercado $\left(\mathrm{R} \$ \mathrm{~kg}^{-1}\right)$ da aveia-branca, de acordo com o seu PH, convertendo-se os valores para dólar americano ao câmbio de R\$2,85 por US\$ (Tabela 1). A receita líquida foi calculada subtraindo-se o custo do tratamento (custo do fungicida + custo da sua aplicação) da receita bruta. Os custos das aplicações foram calculados com base nas equações descritas por MATUO (1990) e FUNDAÇÃO ABC (2003), e os preços dos fungicidas foram aqueles praticados na região de Passo Fundo - RS, na época da colheita da aveia. O resultado econômico foi calculado a partir da diferença entre a receita líquida das parcelas tratadas e a receita líquida na testemunha sem aplicação de fungicida.

As variáveis-controle da ferrugem da folha da aveia, massa do hectolitro, massa de mil grãos, rendimento de grãos e resultado econômico foram submetidas à análise de variância e à comparação de médias, pelo teste de Tukey, a 5\% de probabilidade. 
TABELA 1. Preço médio de comércio da aveia-branca (R\$), de acordo com a massa do hectolitro (PH) praticado na região de Passo Fundo - RS, no mês de janeiro de 2004.

\begin{tabular}{cc}
\hline $\mathrm{PH}\left(\mathrm{g} 100 \mathrm{~L}^{-1}\right)$ & Preço da aveia-branca $\left(\mathrm{US} \$ \mathrm{~kg}^{-1}\right)$ \\
\hline$>39$ & 0,063 \\
$40-49$ & 0,070 \\
$>50$ & 0,074 \\
\hline
\end{tabular}

\section{RESULTADOS E DISCUSSÃO}

$\mathrm{O}$ controle da ferrugem da folha da aveia respondeu significativamente aos fatores volume e dose, sendo indiferente ao fator fungicida, conforme os valores da probabilidade de $\mathrm{F}$ obtidos da análise de variância do arranjo fatorial. Também foi evidenciada a interação significativa entre dose e volume. O melhor desempenho quanto ao controle (Figuras $1 \mathrm{e} 2$ ) foi obtido quando os dois fungicidas foram aplicados com $200 \mathrm{~L} \mathrm{ha}^{-1}$, concordando com os relatos de SAUER (1999), BOLLER et al. (2001) e de BRAUN et al. (2002).

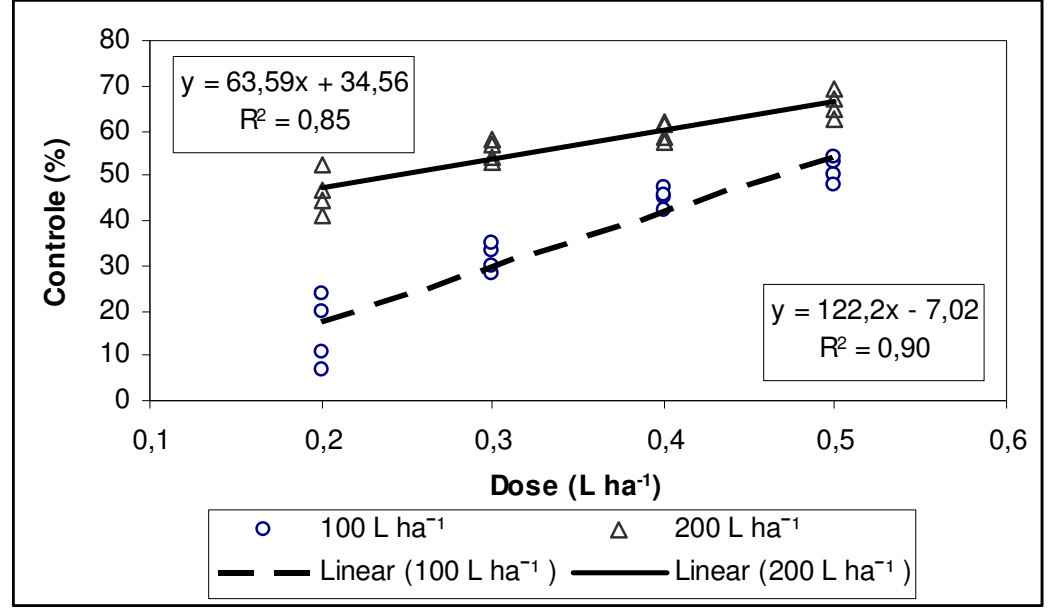

FIGURA 1. Retas ajustadas entre doses do fungicida epoxiconazole + piraclostrobim, aplicado com volumes de 100 e de $200 \mathrm{~L} \mathrm{ha}^{-1}$, e níveis de controle da ferrugem da folha da aveia, cultivar UPFA-20.

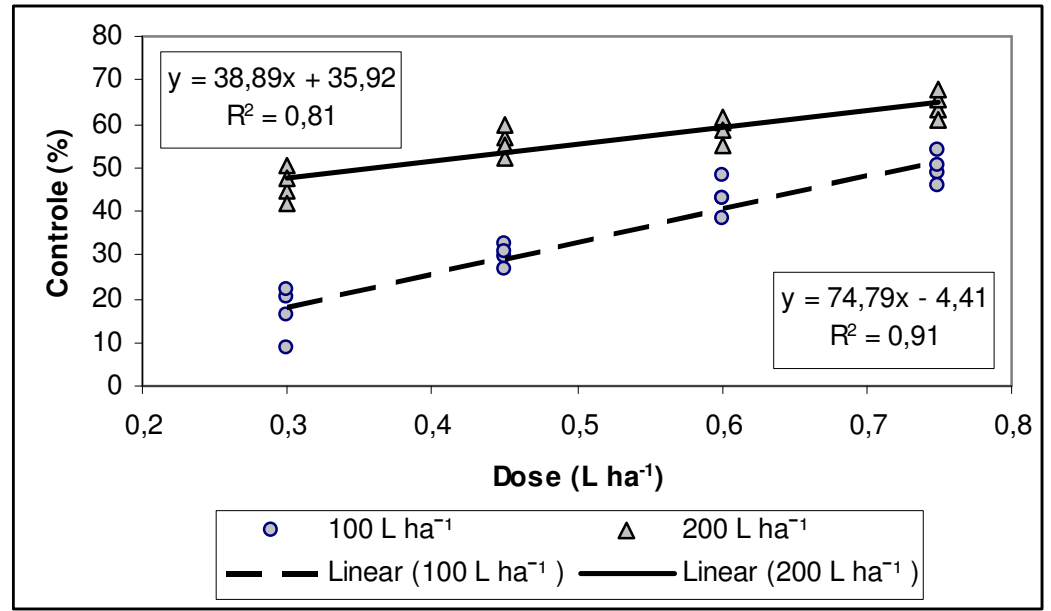

FIGURA 2. Retas ajustadas entre doses do fungicida tebuconazole, aplicado com volumes de 100 e de $200 \mathrm{~L} \mathrm{ha}^{-1}$, e níveis de controle da ferrugem da folha da aveia, cultivar UPFA-20. 
As aplicações dos fungicidas com volume de $200 \mathrm{~L} \mathrm{ha}^{-1}$ e metade das doses recomendadas apresentaram eficácia de controle da ferrugem da folha semelhante às aplicações com $100 \mathrm{~L} \mathrm{ha}^{-1} \mathrm{e}$ as doses recomendadas dos fungicidas. Essa observação indica que o aumento do volume de calda pode contribuir para melhor controle dessa doença ou, até mesmo, para possível redução nas doses dos fungicidas.

A massa do hectolitro (Tabela 2) e a massa de mil grãos (Tabela 3) não apresentaram respostas aos fatores fungicida, dose e volume, porém, na testemunha, sem aplicação de fungicida, ambas as variáveis apresentaram valores significativamente menores aos encontrados nas parcelas tratadas. A melhoria na qualidade dos grãos, em plantas protegidas pelas aplicações de fungicidas, está de acordo com as observações de MARTINELLI (2003), que relatou a redução significativa da qualidade dos grãos de aveia em plantas atacadas pela ferrugem da folha.

TABELA 2. Massa do hectolitro $\left(\mathrm{kg} \mathrm{HL}^{-1}\right)$ de grãos de aveia, cultivar UPFA 20, em função de dois fungicidas aplicados com dois volumes de calda e quatro doses.

\begin{tabular}{ccccc}
\hline \multirow{2}{*}{ Fungicida $^{\mathrm{ns}}$} & $\begin{array}{c}\text { Dose } \\
\left(\mathrm{L} \mathrm{ha}^{-1}\right)\end{array}$ & \multicolumn{2}{c}{ Volume de calda } & \multirow{2}{*}{ Média ${ }^{\mathrm{ns}}$} \\
\cline { 3 - 4 } & 0,20 & $500\left(\mathrm{~L} \mathrm{ha}^{-1}\right)$ & $200\left(\mathrm{~L} \mathrm{ha}^{-1}\right)$ & \\
\hline Epoxiconazole & 0,30 & 55,98 & 54,62 & 55,30 \\
+ & 0,40 & 55,93 & 54,61 & 54,96 \\
piraclostrobim & 0,50 & 54,62 & 55,05 & 55,49 \\
& & 55,46 & 55,81 & 55,21 \\
\hline Média $^{\text {ns }}$ & 0,30 & 54,67 & 55,02 & 55,24 \\
\hline \multirow{2}{*}{ Tebuconazole } & 0,45 & 55,67 & 55,30 & 54,99 \\
& 0,60 & 54,90 & 54,51 & 55,09 \\
& 0,75 & 55,35 & 55,92 & 55,41 \\
Média $^{\text {ns }}$ & & 56,33 & 55,84 \\
\hline
\end{tabular}

Massa do hectolitro na testemunha: $52,71 * *$

ns - Diferenças não-significativas a $5 \%$ entre os fungicidas, os volumes e as doses. ** - Média na testemunha significativamente inferior ao valor de qualquer uma das parcelas tratadas (teste de Tukey a $5 \%$ de probabilidade).

TABELA 3. Massa de mil grãos (g) de aveia, cultivar UPFA 20, em função de dois fungicidas aplicados com dois volumes de calda e quatro doses.

\begin{tabular}{|c|c|c|c|c|}
\hline \multirow{2}{*}{ Fungicida $^{\text {ns }}$} & \multirow{2}{*}{$\begin{array}{c}\text { Dose } \\
\left(\mathrm{L} \mathrm{ha}^{-1}\right)\end{array}$} & \multicolumn{2}{|c|}{ Volume de calda } & \multirow{2}{*}{ Média ${ }^{\mathrm{ns}}$} \\
\hline & & $100\left(\mathrm{~L} \mathrm{ha}^{-1}\right)$ & $200\left(\mathrm{~L} \mathrm{ha}^{-1}\right)$ & \\
\hline Epoxiconazole & 0,20 & 38,35 & 39,30 & 38,82 \\
\hline+ & 0,30 & 40,27 & 38,82 & 39,55 \\
\hline \multirow[t]{2}{*}{ piraclostrobim } & 0,40 & 40,17 & 40,97 & 40,57 \\
\hline & 0,50 & 39,45 & 39,85 & 39,65 \\
\hline \multirow[t]{2}{*}{ Média $^{\text {ns }}$} & & 39,56 & 39,73 & 39,64 \\
\hline & 0,30 & 40,17 & 39,50 & 39,83 \\
\hline \multirow[t]{3}{*}{ Tebuconazole } & 0,45 & 39,65 & 38,45 & 39,05 \\
\hline & 0,60 & 38,50 & 38,37 & 38,43 \\
\hline & 0,75 & 39,57 & 38,80 & 39,18 \\
\hline Média $^{\text {ns }}$ & & 39,47 & 38,78 & 39,12 \\
\hline \multicolumn{5}{|c|}{ Massa de mil grãos na testemunha: $33,40 \mathrm{~g} * *$} \\
\hline
\end{tabular}


Apesar de não se detectarem diferenças significativas entre os tratamentos, as aplicações dos fungicidas favoreceram o desempenho da cultura da aveia, proporcionando acréscimo significativo no rendimento de grãos $\left(\mathrm{kg} \mathrm{ha}^{-1}\right)$ em relação à testemunha (Tabela 4), em 54,78\% para epoxiconazole + piraclostrobim e 58,54\% para o fungicida tebuconazole.

TABELA 4. Rendimento de grãos $\left(\mathrm{kg} \mathrm{ha}^{-1}\right)$ de aveia, cultivar UPFA-20, em função de dois fungicidas aplicados com dois volumes de calda e quatro doses.

\begin{tabular}{|c|c|c|c|c|}
\hline \multirow{2}{*}{ Fungicida $^{\text {ns }}$} & \multirow{2}{*}{$\begin{array}{c}\text { Dose } \\
\left(\mathrm{L} \mathrm{ha}^{-1}\right)\end{array}$} & \multicolumn{2}{|c|}{ Volume de calda } & \multirow{2}{*}{ Média $^{\text {ns }}$} \\
\hline & & $100\left(\mathrm{~L} \mathrm{ha}^{-1}\right)$ & $200\left(\mathrm{~L} \mathrm{ha}^{-1}\right)$ & \\
\hline Epoxiconazole & 0,20 & 3.570 & 3.290 & 3.430 \\
\hline+ & 0,30 & 3.762 & 3.651 & 3.706 \\
\hline \multirow[t]{2}{*}{ piraclostrobim } & 0,40 & 3.537 & 3.863 & 3.700 \\
\hline & 0,50 & 3.673 & 3.688 & 3.680 \\
\hline \multirow[t]{2}{*}{ Média $^{\mathrm{ns}}$} & & 3.635 & 3.623 & 3.629 \\
\hline & 0,30 & 3.706 & 3.592 & 3.649 \\
\hline \multirow[t]{3}{*}{ Tebuconazole } & 0,45 & 3.591 & 3.503 & 3.547 \\
\hline & 0,60 & 3.584 & 3.420 & 3.502 \\
\hline & 0,75 & 3.284 & 3.608 & 3.446 \\
\hline Média $^{\mathrm{ns}}$ & & 3.541 & 3.531 & 3.536 \\
\hline
\end{tabular}

Rendimento de grãos na testemunha: $2.618 \mathrm{~kg} \mathrm{ha}^{-1} * *$

ns - Diferenças não-significativas a 5\% entre os fungicidas, os volumes e as doses. ** - Média na testemunha significativamente inferior ao valor de qualquer uma das parcelas tratadas (teste de Tukey a 5\%de probabilidade).

A análise econômica revelou que as aplicações do fungicida epoxiconazole + piraclostrobim proporcionaram maior resultado econômico quando comparadas ao fungicida tebuconazole (Tabela 5). As interações entre fungicidas e doses foram significativas. A variação da dose do fungicida epoxiconazole + piraclostrobim não implicou diferenças significativas, ao passo que, para o fungicida tebuconazole, as maiores doses apresentaram os menores resultados econômicos, chegando a dose cheia a apresentar resultado econômico negativo (custo do tratamento maior do que o benefício decorrente do controle da doença). Verificou-se, ainda, que o resultado econômico foi independente do volume de calda utilizado. Essas observações demonstram ser oportuno o estudo da redução da dose do fungicida tebuconazole para o controle da ferrugem da folha da aveia, podendo-se melhorar sua relação custo/benefício.

TABELA 5. Resultado econômico (US\$ ha ${ }^{-1}$ ) de dois fungicidas, em quatro doses, aplicados com dois volumes de calda, em aveia cultivar UPFA 20, para o controle da ferrugem da folha.

\begin{tabular}{ccccc}
\hline \multirow{2}{*}{ Fungicida ** } & $\begin{array}{c}\text { Dose } \\
\left(\mathrm{L} \mathrm{ha}^{-1}\right)\end{array}$ & \multicolumn{2}{c}{ Volume de Calda } & \multirow{2}{*}{ Média } \\
\cline { 3 - 4 } & 0,20 & 51,06 & $200\left(\mathrm{~L} \mathrm{ha} \mathrm{h}^{-1}\right)$ & $39,23 \mathrm{~A}$ \\
Epoxiconazole & 0,30 & 57,45 & 27,40 & $52,13 \mathrm{~A}$ \\
+ & 0,40 & 31,66 & 46,81 & $43,22 \mathrm{~A}$ \\
piraclostrobim & 0,50 & 33,73 & 54,78 & $33,30 \mathrm{~A}$ \\
\hline Média $^{\mathrm{ns}}$ & & 43,47 & 32,87 & 41,97 \\
\hline & 0,30 & 54,82 & 40,47 & $49,40 \mathrm{a}$ \\
Tebuconazole $^{*}$ & 0,45 & 34,15 & 43,98 & $29,74 \mathrm{ab}$ \\
& 0,60 & 21,84 & 25,34 & $14,49 \mathrm{~b}$ \\
& 0,75 & $-13,13$ & 7,14 & $-1,65 \mathrm{~b}$ \\
\hline Média $^{\mathrm{n}}$ & & 24,42 & 9,83 & 22,99
\end{tabular}

** Diferenças significativas entre os dois fungicidas, pelo teste de Tukey, a 5\% de probabilidade. Médias seguidas pelas mesmas letras, dentro do mesmo fungicida, não diferem significativamente entre si, pelo teste de Tukey, a $5 \%$ de probabilidade. ${ }^{\text {ns }}$ Diferenças não-significativas a $5 \%$ entre os volumes de calda. 


\section{CONCLUSÕES}

A utilização de maior volume de calda na aplicação de ambos os fungicidas implica maiores níveis de controle da ferrugem da folha da aveia.

Sob a ótica do controle da ferrugem da folha da aveia, o aumento do volume de calda, combinado com a redução da dose dos fungicidas, proporciona resultados equivalentes à utilização da dose recomendada com volume reduzido.

Mesmo os níveis de controle mais reduzidos da ferrugem da folha, obtidos em alguns tratamentos, proporcionam efeitos positivos na qualidade e no rendimento de grãos de aveia.

O resultado econômico depende do fungicida e independe do volume de calda, ao passo que os efeitos das doses dependem do fungicida.

Com a utilização do fungicida epoxiconazole + piraclostrobim, o resultado econômico independe da dose aplicada, enquanto, para o tebuconazole, o aumento dessa implica redução da relação benefício/custo.

\section{REFERÊNCIAS}

AZEVEDO, L.A.S. Qualidade da aplicação de fungicidas protetores. In: protetores: fundamentos para o uso racional. Campinas: Camopi, 2003. p 121-32.

Fungicidas

BOLLER, W.; FORCELINI, C.A.; BRAUN, E. Efeitos de volumes de calda sobre o controle químico de ferrugem da folha e rendimento de grãos da aveia-branca. In: REUNIÃO DA COMISSÃO BRASILEIRA DE PESQUISA DE AVEIA, 21., 2001, Lages. Resultados experimentais... Lages: UDESC, 2001. p.363-5.

BRAUN, E.; BOLLER, W.; FORCELINI, C. A. Controle da ferrugem da folha da aveia: efeitos de volumes de calda nas aplicações de fungicida. In: REUNIÃO DA COMISSÃO BRASILEIRA DE PESQUISA DE AVEIA, 22., 2002, Passo Fundo. Resultados experimentais... Passo Fundo: UPF, 2002. p.457-9.

CALPOUZOS, L.; ROLFS, A.P.; MADSON, M.E.; MARTIN, F.B.; WELSH, J.R.; WILCOXSON, R.D. A new model to measure yield losses caused by stem rust in spring wheat. Agricultural Experiment Station University of Minnesota, 1976. 23 p. (Technical Bulletin, 307).

DELGADO, L.M. Tecnologia para la aplicación de fitosanitarios. Madrid: Universidad Politécnica de Madrid, 1999. 26 p.

FUNDAÇÃO ABC. Custos da mecanização agrícola. Informativo da Fundação ABC, Castro, v.22, p.50-1, 2003.

GUEDES, J.V.C.; DORNELLES, S.H.B. As diversas missões em tecnologia e segurança na aplicação de agrotóxicos. In: Tecnologia e segurança na aplicação de agrotóxicos: novas tecnologias.

Santa Maria: Departamento de Defesa Fitossanitária; Sociedade de Agronomia de Santa Maria, 1998. p.9-15

MARTINELI, J.A. Manejo integrado de doenças da aveia. Fitopatologia Brasileira, Brasília, v.28, n.1, p.98, 2003.

MATUO, T. Técnicas de aplicação de defensivos agrícolas. Jaboticabal: FUNEP, 1990. 139 p.

MATUO, T. Fundamentos da tecnologia de aplicação de agrotóxicos. In: GUEDES, J.V.C.;

DORNELLES, S.H.B. Tecnologia e segurança na aplicação de agrotóxicos: novas tecnologias. Santa Maria: Departamento de Defesa Fitossanitária; Sociedade de Agronomia de Santa Maria, 1998. p.95105. 
OZEKI, Y.; KUNZ, R.P. Manual de aplicação aérea. São Paulo: Ciba Agro, 1994. 46 p.

OZEKI, Y.; KUNZ, R.P. Tecnologia de aplicação aérea - aspectos práticos. In: GUEDES, J.V.C.; DORNELLES, S.H.B. Tecnologia e segurança na aplicação de agrotóxicos: novas tecnologias.

Santa Maria: Departamento de Defesa Fitossanitária; Sociedade de Agronomia de Santa Maria, 1998. p.65-78.

PICININI, E.C.; FERNANDES, J.M. Eficácia de fungicidas no controle da ferrugem da folha da aveia. Fitopatologia Brasileira, Brasília, v.19, n.1, p.74-8, 1994.

SAUER, R. Fungizide brauchen “ziehlwasser”. DLL-Agrarmagazin, München, v.2, n.1, p 76-7, 1999.

SPRAYING SYSTEMS CORPORATION. Teejet Spray Products: Guia do comprador 2002-BR/P. Wheaton, 2003. $36 \mathrm{p}$. 